\title{
A NOTE ON HAAR-LIKE MEASURE FOR GROUP-EXTREMAL SEMIGROUPS
}

\section{MICHAEL FRIEDBERG}

Introduction. If $S$ is a compact group-extremal affine semigroup, it is natural to ask how much of the group structure of the extreme points carries over to $S$. In [4], the author shows it is possible to extend sufficiently many unitary representations to separate points of $S$; in the abelian case, this leads to sufficiently many affine semicharacters to separate points of $S$. We investigate in this note the possibility of extending Haar measure to $S$. Using results in [1], [2], and [3], it can be seen that a compact affine semigroup which supports an idempotent measure must be of the form $X \times Y$ where $X$ and $Y$ are compact convex sets and multiplication is given by $(x, y)$ $\cdot\left(x^{\prime}, y^{\prime}\right)=\left(x, y^{\prime}\right)$. Thus, one cannot hope in general to extend Haar measure and retain all of its properties. However, we will show that if $S$ is a compact group-extremal affine semigroup, then there is a probability measure $\mu$ supported on $S$ (i.e. $\mu$-measure of each nonvoid open set is positive) satisfying

$$
\int f(x y) d \mu(x)=\int f(y x) d \mu(x)=\int f(x) d \mu(x)
$$

for each $y \in S$ and each affine continuous function on $S$.

Preliminaries. If $S$ is a compact Hausdorff space, $C(S)$ will denote the Banach space of continuous complex-valued functions on $S$ with the supremum norm, and $M(S)$ the Banach space of complex-valued regular Borel measures on $S$ with variation norm. We use the notation $\mu(f)=\int f d \mu$ for $f \in C(S), \mu \in M(S)$, and shall employ the RieszKakutani Theorem of ten without comment. If $\mu \in M(S), \mu \geqq 0, C(\mu)$ denotes the complement of the largest open set having $\mu$-measure 0 ; we have $\mu(V)>0$ if and only if $V \cap C(\mu) \neq \varnothing$ holding for each open set $V$ in $S . P(S)$ denotes the subset of $M(S)$ consisting of all nonnegative measures of norm 1 .

Lemma 1. Let $S$ and $K$ be compact Hausdorff spaces and $f: S \rightarrow K$ a continuous function. For $\mu \in P(S)$, define $\left[f^{*}(\mu)\right](g)=\int g(f(x)) d \mu(x)$ for $g \in C(K)$, then:

(a) $f^{*}(\mu) \in P(K)$,

(b) $C\left(f^{*}(\mu)\right)=f(C(\mu))$.

Received by the editors December 12, 1965. 
Proof. (a) is clear. Suppose $x_{0} \notin f(C(\mu))$; there is a function $g \in C(K), 0 \leqq g \leqq 1$ where $g(y)>\frac{1}{2}$ on some open set $V$ containing $x_{0}$, and $g(y) \equiv 0$ for $y \in f(C(\mu))$. We have

$$
\begin{aligned}
0 & =\int_{C(\mu)} g(f(y)) d \mu(y)=\left[f^{*}(\mu)\right](g)=\int g d f^{*}(\mu) \\
& \geqq \int_{V} g d f^{*}(\mu) \geqq \frac{1}{2}\left[f^{*}(\mu)\right](V) .
\end{aligned}
$$

Thus, $\left[f^{*}(\mu)\right](V)=0$ and $V \cap C\left(f^{*}(\mu)\right)=\varnothing$ so that $x_{0} \notin C\left(f^{*}(\mu)\right)$. Conversely, let $x_{0}=f\left(y_{0}\right)$ where $y_{0} \in C(\mu)$ and suppose $x_{0} \notin C\left(f^{*}(\mu)\right)$. There is a function $g \in C(K) 0 \leqq g \leqq 1$ and an open set $V$ containing $y_{0}$ such that $g(f(y))>\frac{1}{2}$ for $y \in V$ while $g(z)=0$ for $z \in C\left(f^{*}(\mu)\right)$. Thus $\left[f^{*}(\mu)\right](g)=0$; however,

$$
\left[f^{*}(\mu)\right](g)=\int g(f(y)) d \mu(y) \geqq \int_{V} g(f(y)) d \mu(y) \geqq \frac{1}{2} \mu(V)>0
$$

since $y_{0} \in V \cap C(\mu)$. With this contradiction, the proof is complete.

Lemma 2. Let $S$ be compact, Hausdorff and $\mu_{i} \in P(S)$ for $i=1,2, \cdots$. Then

$$
\lim _{n} \sum_{i=1}^{n} \frac{1}{2^{i}} \mu_{i}=\mu_{0} \in P(S)
$$

and

$$
C\left(\mu_{0}\right)=\mathrm{Cl} \bigcup_{i=1}^{\infty} C\left(\mu_{i}\right)
$$

(The limit is in variation norm.)

The proof consists of computations similar to those in Lemma 1. so we omit them. We point out, however, that Lemma 2 generalizes the well-known fact that a compact separable Hausdorff space supports a measure; this is done by taking $\mu_{i}$ to be a point mass concentrated at an element of a countable dense subset.

THEOREM 1. Let $S$ be a compact convex subset of a locally convex linear space, and $\mu \in P(S)$. Suppose that $C(\mu)$ contains the extreme points of $S$; then there is a measure $\nu \in P(S)$ satisfying (a) $C(\nu)=S$ and (b) $\int f d \nu=\int f d \mu$ for each continuous affine complex-valued function $f$ on $S$. 
Proof. Fix $n \geqq 1$ and define:

$$
A_{n}=\left\{\left(\lambda_{1}, \cdots, \lambda_{n}\right) \in E^{n}: \lambda_{i} \geqq 0, \sum_{i=1}^{n} \lambda_{i}=1\right\} .
$$

Further, let $m_{n} \in P\left(A_{n}\right)$ such that $C\left(m_{n}\right)=A_{n}$. Let

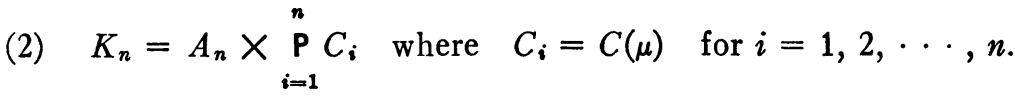

Finally, let $\nu_{n} \in P\left(K_{n}\right)$ be defined by

$$
\nu_{n}(f)=\iint \cdots \int f\left(z, x_{1}, \cdots, x_{n}\right) d m_{n}(z) d \mu\left(x_{1}\right) \cdots d \mu\left(x_{n}\right),
$$

where $f \in C\left(K_{n}\right)$; then $C\left(\nu_{n}\right)=K_{n}$. The function $h_{n}: K_{n} \rightarrow S$ defined by

$$
h_{n}\left\{\left(\lambda_{1}, \cdots, \lambda_{n}\right), x_{1}, \cdots, x_{n}\right\}=\sum_{i=1}^{n} \lambda_{i} x_{i}
$$

is clearly continuous. We define $\sigma_{n}=h_{n}^{*}\left(\nu_{n}\right)$ where $h_{n}^{*}\left(\nu_{n}\right)$ is the measure in Lemma 1. Then we have,

$$
\begin{aligned}
C\left(\sigma_{n}\right) & =C\left(h_{n}^{*}\left(\nu_{n}\right)\right)=h_{n}\left(C\left(\nu_{n}\right)\right)=h_{n}\left(K_{n}\right) \\
& =\left\{y \in S: y=\sum_{i=1}^{n} \lambda_{i} x_{i}, \lambda_{i} \geqq 0, \sum_{i=1}^{n} \lambda_{i}=1, x_{i} \in C(\mu)\right\}
\end{aligned}
$$

If $f$ is a continuous affine function on $S$ then

$$
\begin{aligned}
\sigma_{n}(f) & =\left[h_{n}^{*}\left(\nu_{n}\right)\right](f)=\int f\left(h_{n}(x) d \nu_{n}(x)\right) \\
& =\iint \cdots \int f\left(h_{n}\left(z, x_{1} \cdots x_{n}\right)\right) d m_{n}(z) d \mu\left(x_{1}\right) \cdots d \mu\left(x_{n}\right) \\
& =\iint \cdots \int f\left(\sum_{i=1}^{n} \lambda_{i} x_{i}\right) d m_{n}\left(\lambda_{1} \cdots \lambda_{n}\right) d \mu\left(x_{1}\right) \cdots d \mu\left(x_{n}\right) \\
& =\iint \cdots \int \sum_{i=1}^{n} \lambda_{i} f\left(x_{i}\right) d m_{n}\left(\lambda_{1} \cdots \lambda_{n}\right) d \mu\left(x_{1}\right) \cdots d \mu\left(x_{n}\right) \\
& =\int f d \mu .
\end{aligned}
$$

Letting $\mu_{0}=\lim _{n} \sum_{i=1}^{n} \sigma_{i} / 2^{i}$ we have, by Lemma 2 , that 


$$
\begin{aligned}
C\left(\mu_{0}\right) & =\operatorname{Cl}\left(\bigcup_{n=1}^{\infty} C\left(\sigma_{n}\right)\right) \\
& =\operatorname{Cl}\left(\bigcup_{n=1}^{\infty}\left\{y: y=\sum_{i=1}^{n} \lambda_{i} x_{i}, \lambda_{i} \geqq 0, \sum_{i=1}^{n} \lambda_{i}=1, x_{i} \in C(\mu)\right\}=S .\right.
\end{aligned}
$$

The last equality is a combination of $(5)$, the fact that $C(\mu)$ contains the extreme points of $S$, and the Krein-Milman Theorem. Further, for each affine continuous function $f$,

$$
\int f d \mu_{0}=\lim _{n} \sum_{i=1}^{n} \frac{1}{2^{i}} \sigma_{i}(f)=\int f d \mu .
$$

The measure $\mu_{0}$ satisfies properties (a) and (b) of the theorem.

We apply Theorem 1 to the group-extremal affine semigroup $S$ (for definitions see [3]) whose extreme points form the compact group $G$. Let $m$ be Haar measure of the group $G$ extended to the Borel sets of $S$ by the formula $m(E)=m(E \cap G)$. Then $C(m)=G$ and $\int f d m$ $=\int_{G} f(x) d m(x)$ for each continuous function $f$ on $S$. By Theorem 1, we obtain a measure $\mu_{0} \in P(S)$ where $C\left(\mu_{0}\right)=S$ and $\int f d \mu_{0}=\int f d m$ for each affine continuous function $f$. If $f$ is affine continuous on $S$, the function $f^{y}(x) \equiv f(x y)$ is another affine continuous function for each fixed $y \in S$. Thus, for $g \in G \int f(x g) d \mu_{0}(x)=\int f^{o} d \mu_{0}=\int f^{o} d m=\int f d m$ $=\int f(x) d \mu_{0}(x)$. Since $y \rightarrow \int f(x y) d \mu_{0}(x)$ is also affine continuous, we have $\int f(x y) d \mu_{0}(x)=\int f(x) d \mu_{0}(x)$ for every $y \in S$. Similarly, $\int f(y x) d \mu_{0}(x)$ $=\int f(x) d \mu_{0}(x)$ for each $y \in S$. We have proved

THEOREM 2. If $S$ is a compact group-extremal affine semigroup, there is a measure $\mu_{0} \in P(S)$ for which $C\left(\mu_{0}\right)=S$ and $\int f(x y) d \mu_{0}(x)$ $=\int f(y x) d \mu_{0}(x)=\int f(x) d \mu_{0}(x)$ for each $y \in S$ and each affine continuous $f \in C(S)$.

The author would like to express his thanks to Professor R. J. Koch for his comments and advice in the preparation of this note.

\section{BibliogRAPHY}

1. H. S. Collins, Idempotent measures on compact semigroups, Proc. Amer. Math. Soc. 13 (1962), 442-446.

2. - Remarks on affine semigroups, Pacific J. Math. 12 (1962), 449-455.

3. H. S. Collins and H. Cohen, Affine semigroups, Trans. Amer. Math. Soc. 93 (1959), 97-113.

4. M. Friedberg, On representations of certain semigroups, Pacific J. Math. (to appear).

EMORY UNIVERSITY 